\title{
Comentario
}

\section{ARGENTINA, CHILE Y UN ACUERDO DE ÚLTIMA GENERACIÓN}

\author{
Nicolás Nieto* \\ Irene Sacco**
}

\section{Resumen}

Desde mayo de 2019, momento de la entrada en vigencia del Sexagésimo Primer Protocolo Adicional entre Argentina y Chile, importantes cambios políticosinstitucionales han acontecido en los ámbitos domésticos y a nivel mundial. La reciente vigencia del acuerdo y la actual retracción global del comercio hacen que los datos en materia de exportación e importación no hayan experimentado variaciones importantes. En este artículo se analiza el desarrollo de la negociación del acuerdo; sus capítulos más relevantes; los datos arrojados por las estadísticas oficiales de Argentina y Chile. Hacia el final, se trazan algunas ideas para avanzar en las instancias del acuerdo.

Palabras claves: Argentina, Chile, acuerdo comercial, acuerdos de nueva generación.

\section{ARGENTINA, CHILE AND A NEW-GENERATION AGREEMENT}

\begin{abstract}
Since May 2019, when the 61st Additional Protocol between Argentina and Chile came into force, important political-institutional changes have taken place at the domestic and global level. The recent validity of the agreement and the current global retraction of trade mean that the data on exports and imports have not experienced significant variations. This article analyzes the development of the negotiation of the agreement; its most relevant chapters; the data produced by the statistics of Argentina and Chile. Towards the end, some ideas are outlined to advance in some instances of the agreement.

\footnotetext{
* Licenciado en Relaciones Internacionales por la Universidad de Rosario (UNR). Integrante de la Cátedra Libre de Chile (UNR). Analista de Comercio Exterior y Desarrollo Productivo en Grupo Rambla (Arroyo Seco, Santa Fe). Rosario, Argentina. Correo Electrónico: nicolas.nadin.nieto@gmail.com. ORCID: 0000-0003-1668-6505.

** Licenciada en Relaciones Internacionales por la Universidad Nacional de Rosario (UNR). Integrante de la Cátedra Libre de Chile (UNR). Miembro del Grupo de Estudios sobre Finanzas Internacionales (Instituto de Investigaciones FCPOLIT-UNR). Asistente Profesional en la Secretaría de Cooperación Internacional e Integración Regional (Provincia de Santa Fe). Rosario, Argentina. Correo electrónico: irenesacco88@gmail.com. ORCID: 0000-0002-2056-0481.
} 
Revista Integración y Cooperación Internacional, no 34, enero-junio 2022, pp. 49-64

ISSN 1852-9798

Key words: Argentina, Chile, trade agreement, new generation agreements

TRABAJO RECIBIDO: 14/10/2021 - TRABAJO ACEPTADO: 10/11/2021 


\section{Introducción}

Movilizaciones ciudadanas, elecciones, cambios de gobiernos y una pandemia. Los dos años de existencia que cumplió el último acuerdo comercial signado entre Argentina y Chile, fueron todo menos aburridos. Desde su entrada en vigencia, acontecimientos tan particulares como relevantes sobrevinieron y marcaron el contexto nacional, regional e internacional de los actores, obligándolos a actuar en consecuencia.

En el presente artículo se intenta analizar no sólo el tratado a través de sus capítulos, sino también elimpacto que tuvo en la relación comercial y el contexto donde se desarrolló su proceso de negociación. De esta manera, el objetivo que se propone es develar si este último, con sus particularidades, ha logrado profundizar el vínculo en términos nominales y de valor agregado pese alescenario en el cual le tocó desarrollarse.

Para ello, el recorrido inicia desde un nivel más general hacia uno más específico, comenzando por describir el contexto internacional, regional y nacional en el cual interactuaron Argentina y Chile. Seguidamente, se detallan los apartados más importantes del tratado comercial, destacando aquellas que resultaron más innovadoras. A continuación, se presentan datos con respecto al desempeño comercial que ambos países signatarios tuvieron previa y subsiguientemente al acuerdo. Por último, se realiza un análisis sobre el escenario posterior, donde se intenta contextualizar los números presentados en el apartado anterior y destacar aspectos más allá de lo netamente cuantitativo.

\section{Contexto internacional: la pandemia}

La celeridad con la cual se expandió el nuevo coronavirus por el mundo hizo que toda cuestión más allá de la situación sanitaria quedara relegada a un segundo plano. Pese a la divergencia de las estrategias emprendidas por las diferentes naciones, las consecuencias económicas fueron implacables. En cuestión de meses, la enfermedad detectada por primera vez en la ciudad de Wuhan logró dar la vuelta al mundo. Lo precipitado de la situación obligó a ensayar medidas poco planificadas. Nuestra civilización caía presa de la globalización que supo erigir.

Lo disruptivo de la situación se manifestó a través del parate al cual debió someterse la economía mundial con el propósito de disminuir la circulación del virus. Ciudades otrora repletas de gente y de tráfico ajetreado se transformaron en desiertos de concreto. La naturaleza retomó el control de lo que históricamente le ha pertenecido (al menos momentáneamente). No obstante, el nuevo coronavirus más que interrumpir las propias dinámicas del orden político-económico liberal, no hizo más que acelerarlas. Como sostienen los autores Actis y Creus, "la pandemia no fue tanto la causa que puso al mundo patas para arriba, sino más bien el gatillo" (Actis \& Creus, 2020, 273).

El crecimiento anémico de la economía global, la desigualdad acuciante y grosera en la distribución de la riqueza, la desilusión de clase media en los países desarrollados con respecto al desempeño de la economía, la crisis de las democracias liberales producto de la exigua credibilidad de los partidos políticos tradicionales frente 
a la ciudadanía; son procesos de los cuales se tenía conocimiento pero que son difíciles de abordar por la complejidad que implican. La pandemia no hizo más que revelarlos de una manera brusca. Las potencias occidentales, otrora paladines del desarrollo, ven con recelo como el centro del dinamismo económico mundial se traslada con rapidez al pacífico. Asia, y principalmente China, ya no sólo provee mano de obra barata. La innovación dejó de ser algo monopolizado por Europa y Estados Unidos (EEUU). A esto debe sumarse el acelerado cambio tecnológico que obliga a numerosos sectores de la sociedad a innovarse o quedar relegados.

Frente a este escenario, la ciudadanía en occidente está enojada. Se siente defraudada por las consecuencias de una globalización que sus gobiernos no dudaron en promover. El estándar de vida que supieron alcanzar en las últimas décadas comienza a verse amenazado frente a una economía mundial cada vez más integrada, pero en muchas ocasiones despiadada. Atender este malestar se torna sumamente necesario para las autoridades occidentales si no quieren tensar aún más la delicada relación con su electorado. Una representatividad endeble anima las pasiones, ahonda las diferencias y promueve las posiciones extremas. El hecho que hayan aparecido figuras o partidos contra la globalización que buscan en el inmigrante un chivo expiatorio para expiar culpas, no hace más que demostrarlo.

\section{Contexto nacional: cambio de gobierno y movilización ciudadana}

En lo que se refiere a Chile, la irrupción de las movilizaciones de octubre y noviembre de 2019 en las calles de Santiago hizo tambalear los propios cimientos de toda la sociedad chilena. La clase gobernante, acusada por los partícipes de haberse desentendido de los intereses y problemas de la ciudadanía, tiene la ardua tarea de recomponer los lazos con una sociedad desilusionada con los partidos políticos tradicionales chilenos.

El proceso tomó tamaña trascendencia que terminó con la constitución chilena de 1980. A mediados de mayo del presente año, se eligieron a quienes conformarán la Convención Constituyente. La misma, está conformada por igual cantidad de hombres y mujeres (algo inédito en el mundo) y con la participación de representantes de pueblos originarios (históricamente relegados por el establishment político chileno). Además, se realizaron las primeras elecciones de gobernadores regionales del país, cargos que reemplazaron a los intendentes regionales quienes históricamente eran designados por el poder ejecutivo de turno en Santiago. Esto último se entiende dentro de un proceso de descentralización del Estado nacional chileno, donde se intenta otorgar mayores atribuciones a las regiones como divisiones territoriales.

De esta manera, 2021 es el año que marca el inicio de una nueva era en Chile. Deberá transitar un período de grandes transformaciones tendientes a remediar las consecuencias del modelo neoliberal. Atendiendo la gran desigualdad y el desentendimiento del Estado con los servicios sociales básicos (principales responsables de las movilizaciones de 2019).

En Argentina, las elecciones generales de 2019 marcaron el regreso de la coalición de gobierno del "Frente de Todos", alianza rival del entonces presidente Mauricio Macri y que aglutinó a amplios sectores del peronismo (destacándose el 
kirchnerismo entre ellos). En un país acostumbrado a la carencia de políticas públicas a largo plazo, todo cambio de gobierno despierta una gran expectativa e incertidumbre. Castigada por la recesión económica de los últimos años y acorralada por las obligaciones de una deuda externa que creció durante la administración macrista, el nuevo mandatario Alberto Fernández se enfrentó a una economía deprimida, con inflación endémica, déficit fiscal persistente y crecimiento de la pobreza. El desencadenamiento de la pandemia en 2020 encontró a Argentina en una posición comprometida, lo que profundizó aún más su aguda crisis económica.

\section{Escenario regional y bilateral.}

En cuanto al ámbito regional, merece un detenido análisis de unos años anteriores al momento contemporáneo. El triunfo de Mauricio Macri en Argentina en 2015 marcaría el inicio de una serie de derrotas de gobiernos de centro izquierda en Sudamérica. La destitución de Dilma Rousseff en Brasil en 2016 (que conduciría a la victoria de Jair Bolsonaro), el regreso al poder en 2018 de Sebastián Piñera en Chile y la retirada de Evo Morales de la presidencia boliviana, tras ser obligado a renunciar por el golpe militar de 2019, son testigos de todo ello. Estos acontecimientos marcaron el arribo de gobiernos de signos opuestos y fuertemente críticos a sus antecesores.

Con respecto a las relaciones exteriores, la llegada de Mauricio Macri a la Casa Rosada representó un punto de inflexión en Argentina. Afín a políticas aperturistas que promuevan el comercio internacional con otros países, el mandatario priorizó la firma de acuerdos comerciales como un capítulo importante dentro su gestión. La promoción de un Tratado de Libre Comercio (TLC) entre el Mercosur y la Unión Europea, donde Argentina pasó de tener una posición defensiva a transformarse en su principal promotor, es un ejemplo de ello. En coincidencia con lo anterior, la conclusión de un nuevo acuerdo comercial con Chile se convirtió rápidamente en un objetivo a cumplir. Además, la coincidencia político ideológica y la relación personal que unía a Mauricio Macri y Sebastián Piñera, no hizo más que facilitar el proceso de negociación y su posterior aprobación.

No obstante, como se mencionó anteriormente, este escenario se vio interrumpido con la llegada de Alberto Fernández a la presidencia argentina. Es más, el vínculo con su par chileno, Sebastián Piñera, se vio truncado incluso antes de que arribara a la Casa Rosada, cuando criticó el accionar de este último frente a las masivas movilizaciones de finales de 2019. A esto debe sumarse las comparaciones que realizó sobre la situación sanitaria argentina y chilena durante los primeros meses de la actual pandemia, cuando aseguró que el desempeño de las políticas públicas argentinas fue mejor. Estas palabras no tuvieron una buena recepción en los pasillos de La Moneda y provocaron airadas idas y venidas entre las Cancillerías en Santiago y Buenos Aires. Pese a los esfuerzos diplomáticos de ambos lados por encauzar la relación personal entre los dirigentes, la misma nunca trascendió lo ceremonial y protocolar.

Sin embargo, la relación bilateral se vio poco afectada en términos generales. A pesar de la existencia de lo que se podrían considerar algunos malos entendidos, los gobiernos de ambos paísescontinuaron llevando a cabo encuentros y firmando acuerdos. Esto se explica debido a que el vínculo interestatal ha llegado a un punto de coordinación gracias a que ambos Estados lograron erigir un andamiaje institucional 
capaz de sortear posibles altercados entre presidentes o continuos cambios de gobierno. Esto último pudo apreciarse a principios de septiembre del corriente año, luego de que Piñera declarara la ampliación de la plataforma continental chilena en aguas próximas a la Antártida. La veracidad o no de los argumentos esgrimidos desde Santiago para emprender la decisión exceden los fines de este artículo. Lo mismo puede decirse de los motivos que llevaron al presidente chileno a hacer esto, aunque se cree que la derrota electoral de su partido en las recientes elecciones, así como el deterioro de su imagen tienen una fuerte relación. Lo importante aquí es que pese a la controversia que se originó entre los gobiernos, ambas partes priorizaron el respeto al tratado de 1984 y se comprometieron a resolverla a través del diálogo.

Lo antes expuesto se torna aún más relevante al identificar la metodología de círculos concéntricos elaborada por Miryam Colacrai "que comprenden a los diversos actores que desarrollan acciones en el amplio campo de la política exterior", desde la diplomacia tradicional, el vínculo subnacional (o paradiplomático), los Comités de Integración (espacios transregionales), hasta el "espacio construido por la clase política, o parte de ella" en referencia al vínculo entre partidos políticos y sus líderes (Colacrai, 2015, 8).

Para arribar a esta etapa, Argentina y Chile debieron transitar un proceso gradual, no exento de sobresaltos, que se inauguró con la vuelta a la democracia en el cono sur y el Tratado de Paz y Amistad de 1984. Durante el mismo, ambos actores fueron capaces de cambiar paulatinamente la percepción que tenían sobre su vecino y acordar incluso en áreas donde se ven implicados intereses vitales. De no haber existido este cambio, la cooperación se hubiera tornado sumamente difícil de concretar (Nieto, 2019, 68). Lo anterior toma aún mayor relevancia si se recuerda que hace poco más de cuarenta años, desacuerdos fronterizos en torno al Canal de Beagle casi precipitan un conflicto armado abierto entre los Estados.

\section{Un acuerdo última generación}

En el marco de la VIII Reunión Binacional de Ministros y la VI Reunión de Intendentes y Gobernadores de la frontera común celebrada los días 15 y 16 de diciembre de 2016 en la ciudad de Buenos Aires, Argentina y Chile acordaron maximizar el potencial de la relación en materia económica y comercial entre ambos países. Esto se buscó a través de la negociación de un acuerdo comercial que permitiese avanzar en la integración bilateral, estimular las inversiones, fortalecer las cadenas de valor entre ambas partes y promover los mercados para ambos países.

El comunicado final de la reunión binacional de ministros señalaba que la negociación se iniciaría "a la brevedad con la intención de concluir durante el año 2017, incluirá entre otras disciplinas: compras públicas, facilitación de comercio, coherencia regulatoria, política de competencia, servicios, inversiones, comercio electrónico y cooperación” (Declaración VIII Reunión Binacional de Ministros de Argentina y Chile, 2016).

Las partes decidieron profundizar y extender el marco jurídico bilateral del Acuerdo de Complementación Económica N ${ }^{\circ} 16$ del año 1991. Éste se había suscripto en un contexto de armonización del capital/trabajo y de apertura del comercio exterior 
en el que se encontraban empeñados los gobiernos de Miguel Patricio Aylwin en Chile y de Carlos Ménem en Argentina. Entendimiento que era compatible con los propósitos de libre comercio del entonces Acuerdo General sobre Aranceles Aduaneros y Comercio (GATT, por sus siglas en inglés) y de la Asociación Latinoamericana de Integración (ALADI). Era, finalmente, un acuerdo de 16 capítulos con 32 artículos.

El resultado de ese acuerdo era menos complejo como los mismos intercambios lo eran en las últimas décadas del siglo XX. Sin embargo, la sofisticación del comercio y la rápida propagación de nuevos formatos comerciales se desacoplaron de la letra del instrumento jurídico. Empezó a intensificarse lo que Richard Baldwin denomina la brecha entre el comercio del siglo XXI y las normas comerciales del siglo XX (Baldwin, 2011).

Como fuera consignado en los apartados anteriores, durante las administraciones de Macri y Piñera se concluyó que el comercio era sustancialmente más intrincado y comprendía el nexo entre comercio-inversión-servicio que la letra del acuerdo de 1991 ya no reflejaba. Asimismo, se planteaba como un escalón más en la etapa del proceso de integración económica entre Argentina y Chile en base a dos entendimientos vigentes: el Acuerdo de Complementación Económica N ${ }^{\circ} 35$ MERCOSUR-Chile (ACE 35) y el Acuerdo para Evitar la Doble Tributación (en vigencia desde octubre de 2016).

Con ello, el nuevo acuerdo, se basó en estudios de las competencias y nuevas modalidades de las transformaciones en materia de comercio internacional para llegar a negociar sus 20 (veinte) capítulos y 2 (dos) anexos correspondientes a las listas concesionales de Argentina y Chile respectivamente. Cabe recordar que no se trata de un acuerdo arancelario, incluye diversas disciplinas para facilitar el comercio.

Al cabo de la V Ronda de negociaciones, que se desarrolló en la ciudad de Buenos Aires entre los días 25 y 26 de septiembre del 2017, Argentina y Chile culminaron la negociación de la letra del mencionado acuerdo, previendo que su firma no demoraría más de dos semanas luego de la revisiónlegal del texto.

Entre los temas más novedosos se puede destacar el capítulo 3 en materia de "Emprendedores y lasMicro, Pequeñas y Medianas Empresas (MIPyMEs)"; el 4 que refiere a las "Medidas sanitarias y fitosanitarias"; el 7 de "Contratación Pública". Por su parte, los capítulos 9, 10 y 11 se enfocan en "Comercio de Servicios", "Telecomunicaciones" y "Comercio Electrónico".

El capítulo 13 sobre "Comercio y Medio Ambiente" sienta las bases para promover políticas comerciales y ambientales que se apoyen mutuamente. Establece la refuncionalización de las instancias bilaterales existentes a partir del Tratado entre la República Argentina y la República de Chile sobre Medio Ambiente de 1991 como espacio para las negociaciones políticas y prácticas para mejorar los niveles de protección ambiental.

Mientras el capítulo 14 introduce que las "Partes reconocen la importancia de la incorporación de la perspectiva de género en la promoción de un crecimiento económico inclusivo y el rol clave que las políticas de género pueden desempeñar en la consecución de un mayor desarrollo sostenible" (Sexagésimo Primer Protocolo Adicional, 2017, Capítulo 14). 
La letra final del documento pasó a la revisión y procedimiento interno de ratificación de los países firmantes. En diciembre de 2018, la Cámara de Diputados de la República Argentina sancionó el proyecto de ley mediante el cual se aprobó el Protocolo Adicional $\mathrm{N}^{\circ} 61$, tras contar con la aceptación de la Cámara de Senadores en el mes de octubre de ese año. En Chile, la Cámara de Diputados lo aprobó en noviembre de 2018, y en enero del 2019 entró en vigencia con el respaldo de la mayoría del Senado.

\section{Datos cuantitativos de la relación comercial bilateral entre Argentina y Chile}

Ha pasado poco tiempo desde la entrada en vigor del acuerdo. No obstante, es posible formular algunas conclusiones si se observan los datos arrojados por el Instituto de Estadísticas y Censos (INDEC) de Argentina y el Servicio Nacional de Aduana del Gobierno de Chile

El Gráfico 1 demuestra el valor que sumaron mensualmente las exportaciones mutuas de Argentina y Chile entre mayo de 2017 y junio del 2020. El mismo denota como - pese a la entrada en vigor del nuevo acuerdo en mayo de 2019 - las exportaciones no sufrieron un cambio notorio.

En cuanto a la Argentina, el valor de lo exportado al país vecino rondó, en términos generales, entre los 200 millones y 250 millones de dólares. Se destaca el último bimestre de 2018 y diciembre de 2019, donde se alcanzaron valores ampliamente superiores a los 250 millones. Por otra parte, las exportaciones argentinas tuvieron un muy buen desempeño en 2021, logrando superar los 300 millones de dólares. La retracción que sufrieron a partir de marzo del 2020 puede explicarse el contexto del COVID-19, rozando mínimos históricos.

En el caso chileno, se observa la gran diferencia de los valores de sus exportaciones con respecto a su contraparte. Cerca de los 50 millones de dólares, se destacan los meses entre mayo y agosto de 2018 y agosto de 2019 donde se superó holgadamente dicha cifra. Respecto al acuerdo, luego de su entrada en vigor los valores sufrieron una retracción, llegando a bajos inéditos para los tres años analizados. Con el repunte que comienza a partir de mayo de 2020, se alcanzó a superar los 50 millones aunque estuvo lejos de los números de 2018 y 2019 antes mencionados. 
Gráfico 1: Exportaciones de Argentina a Chile y de Chile a Argentina

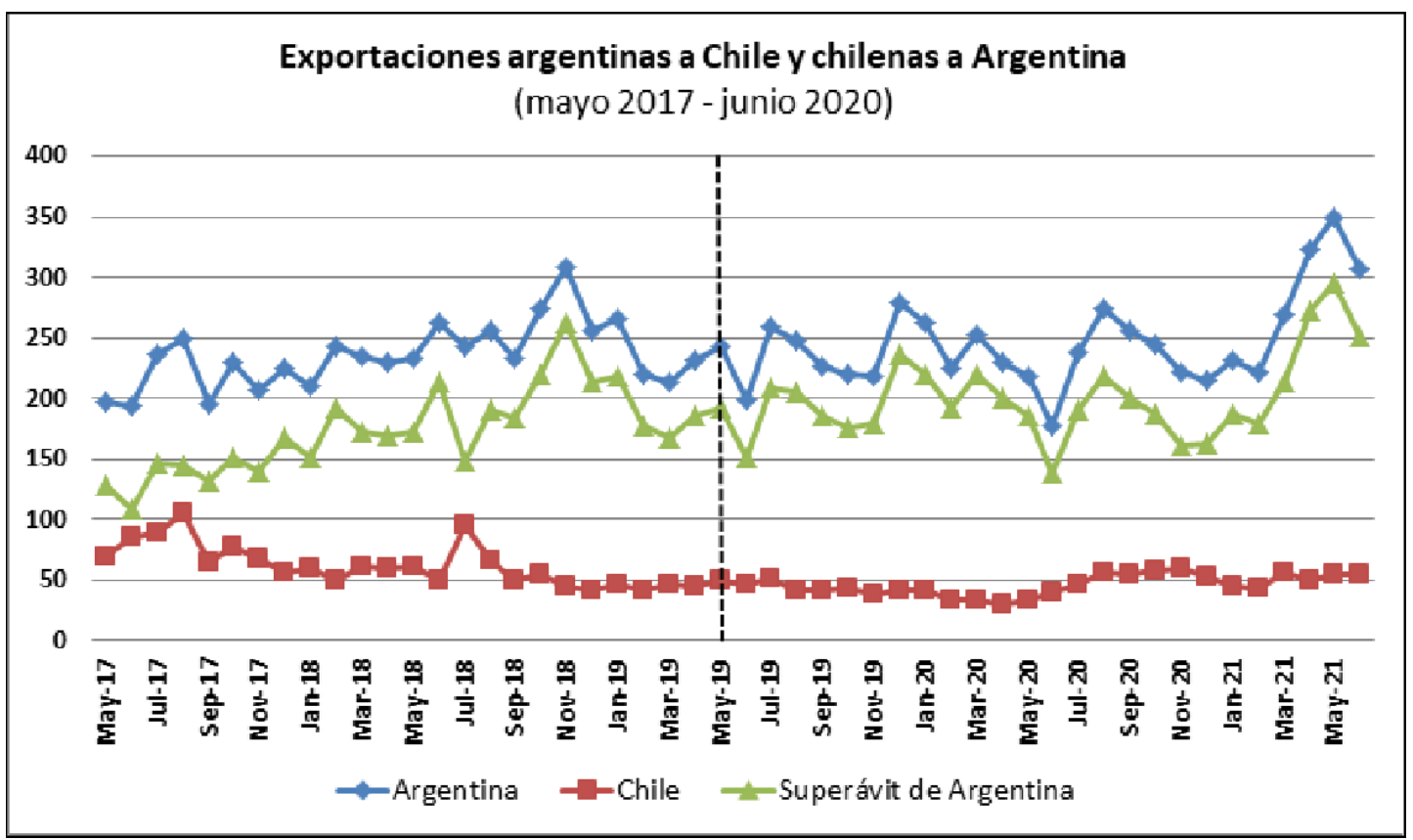

* La línea punteada indica la entrada en vigencia del acuerdo comercial

Fuente: Instituto Nacional de Estadísticas y Censos de Argentina (INDEC)

Asimismo, en el Gráfico 1 puede percibirse la gran divergencia existente entre la suma de las exportaciones argentinas y las chilenas. De esta forma, se puede detectar que en la relación comercial Argentina goza de un superávit persistente. Este último fue incrementándose paulatinamente hasta noviembre de 2018, demostrando un valor superior a los 250 millones de dólares. Esta cifra es debido a que en aquella época, como se mencionó anteriormente, las exportaciones argentinas superaron los 300 millones mientras que las chilenas no llegaron superar los 50. En los meses posteriores, el superávit argentino se retrae a valores inferiores a los 200 millones. Luego de la entrada en vigor del último acuerdo, el mismo fluctúa entre los 150 millones y los 250 millones. Por último, es importante destacar cómo las cifras sufren una caída desde abril a junio del corriente año producto de la caída de las exportaciones argentinas y el leve repunte que sufren las chilenas. No obstante, luego de fluctuar nuevamente, en 2021 comienza a subir y rozar los 300 millones en mayo del corriente año. Por último, denota como las variaciones que sufre este indicador está fuertemente vinculado al desempeño que tuvieron las exportaciones argentinas. Esto último da cuenta nuevamente la poca fluctuación que arrojaron las exportaciones de Chile.

Al hablar sobre la relación comercial entre determinados países, es interesante observar el comportamiento que tuvieron los mismos con respecto a sus principales socios en este ámbito. Contemplar si la retracción o incremento de los valores exportados de Argentina hacia Chile y viceversa son coincidentes con las fluctuaciones que ambos tuvieron en cuanto a su comercio internacional con los demás países, puede contribuir a un análisis más convincente. 
En el Gráfico 2 se muestran los montos que sumaron anualmente las exportaciones argentinas a Chile en comparación con sus principales socios comerciales: Brasil, China y EEUU. En primer lugar es notoria la diferencia entre las cifras arrojadas por las exportaciones que tuvieron destino a Brasil (principal socio comercial de Argentina) con respecto a China (su segundo socio) y más aún con Chile. De esta manera, se observa la dependencia que posee nuestro país con el gigante sudamericano, el cual en los años 2016, 2017 y 2018 duplicó las cifras acaparadas por el mercado asiático; diferencia atenuada en 2019 y 2020.

En cuanto al país trasandino, el mismo se posicionó como el cuarto destino de las exportaciones argentinas, sufriendo un leve incremento entre 2017 y 2019, para volver a retraerse en 2020. Además, es necesario remarcar que las fluctuaciones que sufrieron estas últimas (entre los 2300 millones y los 3000 millones de dólares) fueron menos notorias que la de los demás países. Se destacan los casos de Brasil (que va desde los 8000 millones y los 12000 millones) y China (entre los 4000 millones y los 7000 millones).

\section{Gráfico 2: Exportaciones totales de Argentina a Chile y a sus principales socios comerciales}

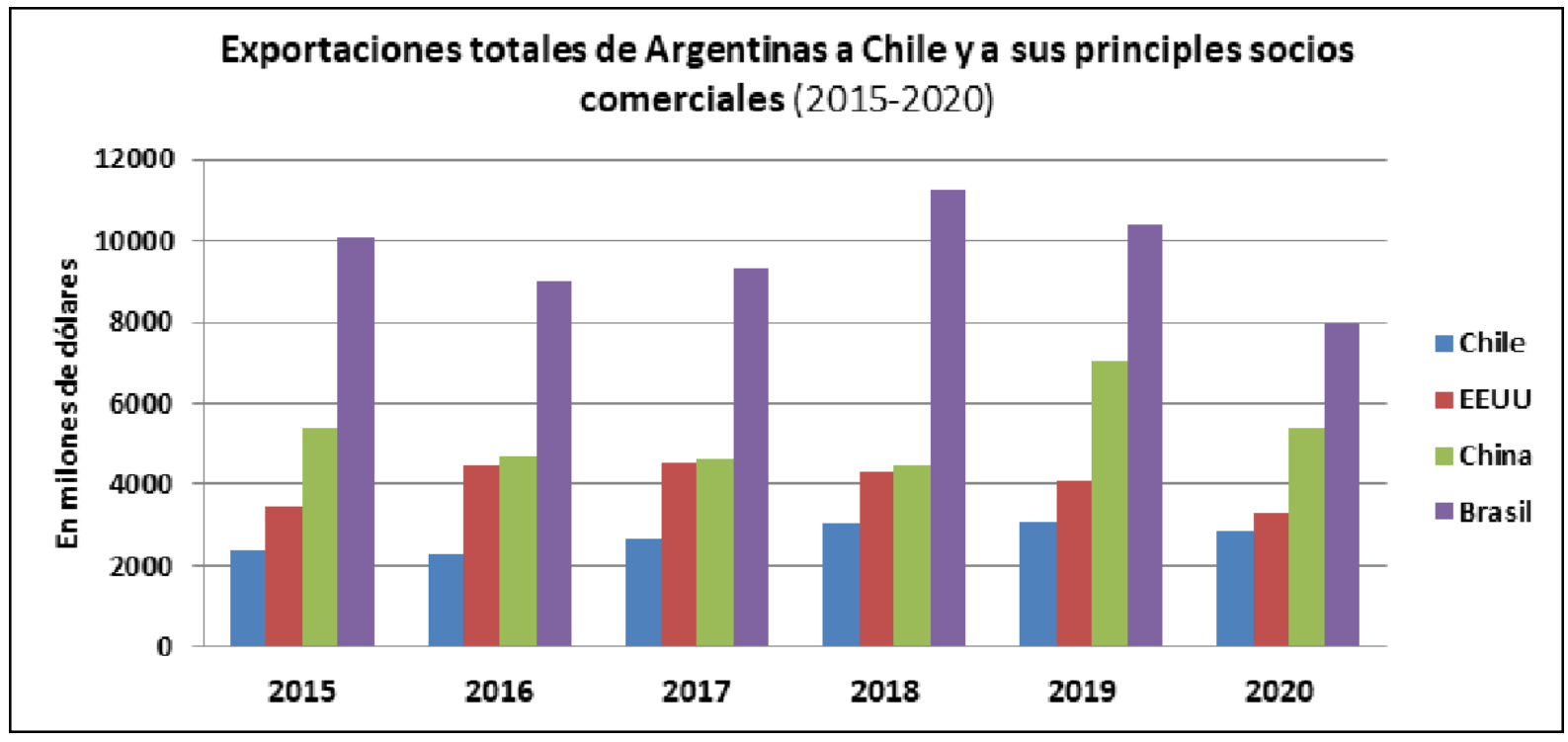

Fuente: Instituto Nacional de Estadística y Censos (INDEC)

En lo que respecta a Chile, el Gráfico 3 compara las sumas anuales que arrojaron las exportaciones que tuvieron como destino a la Argentina con las que acapararon los principales socios comerciales del país vecino. En este caso, se aprecia la primacía que cobró el mercado chino en los años analizados al posicionarse cómodamente como el principal destino comercial. La misma fue aumentando paulatinamente hasta el año 2018, donde superó los 24.000 millones de dólares y duplicó los valores de las exportaciones que se dirigieron a los EEUU. Esto se mantuvo durante el año siguiente, aunque de manera más atenuada. No obstante, en 2020 se superaron los valores de 2018, logrando cifras históricas.

El mercado chino acaparó dos veces y media lo arrojado por el país 
norteamericano. Tamaña dependencia es aún mayor a la de Argentina con respecto a Brasil. Es significativa la diferencia entre la suma de las exportaciones hacia Argentina en comparación con las que se destinaron a Japón (tercer socio comercial de Chile). En el primer caso no superó los 1300 millones en los seis años estudiados, destacándose sólo 2018. Las exportaciones que tuvieron como destino al país insular, semantuvieron por arriba de los 5000 millones, llegando a más de 6700 millones en 2018.

Como se vio en el caso argentino, Chile se posiciona holgadamente como el cuarto destino de las exportaciones argentinas (por detrás de EEUU). Lo anterior no se replica en el caso chileno debido a que incluso en el año 2018 (donde el comercio con Argentina aumentó), nuestro país se posicionó como el decimotercer lugar, muy por detrás de varios países latinoamericanos (Brasil, Perú o México), europeos (Holanda o España) y asiáticos (India, Taiwán o Corea del Sur). Por un lado, esto se explica debido al carácter notoriamente deficitario que posee el vínculo comercial para Chile. Por otro lado, la existencia de tratados comerciales que el Estado chileno posee con las principales economías del mundo y la mayoría de los países latinoamericanos, permite el acceso de la producción chilena a otros mercados que, pese a la distancia geográfica, se tornan más atractivos por la capacidad de consumo de los mismos y/o la complementación económica posible entre las naciones.

Gráfico 3: Exportaciones Totales de Chile a Argentina y a sus principales socios comerciales

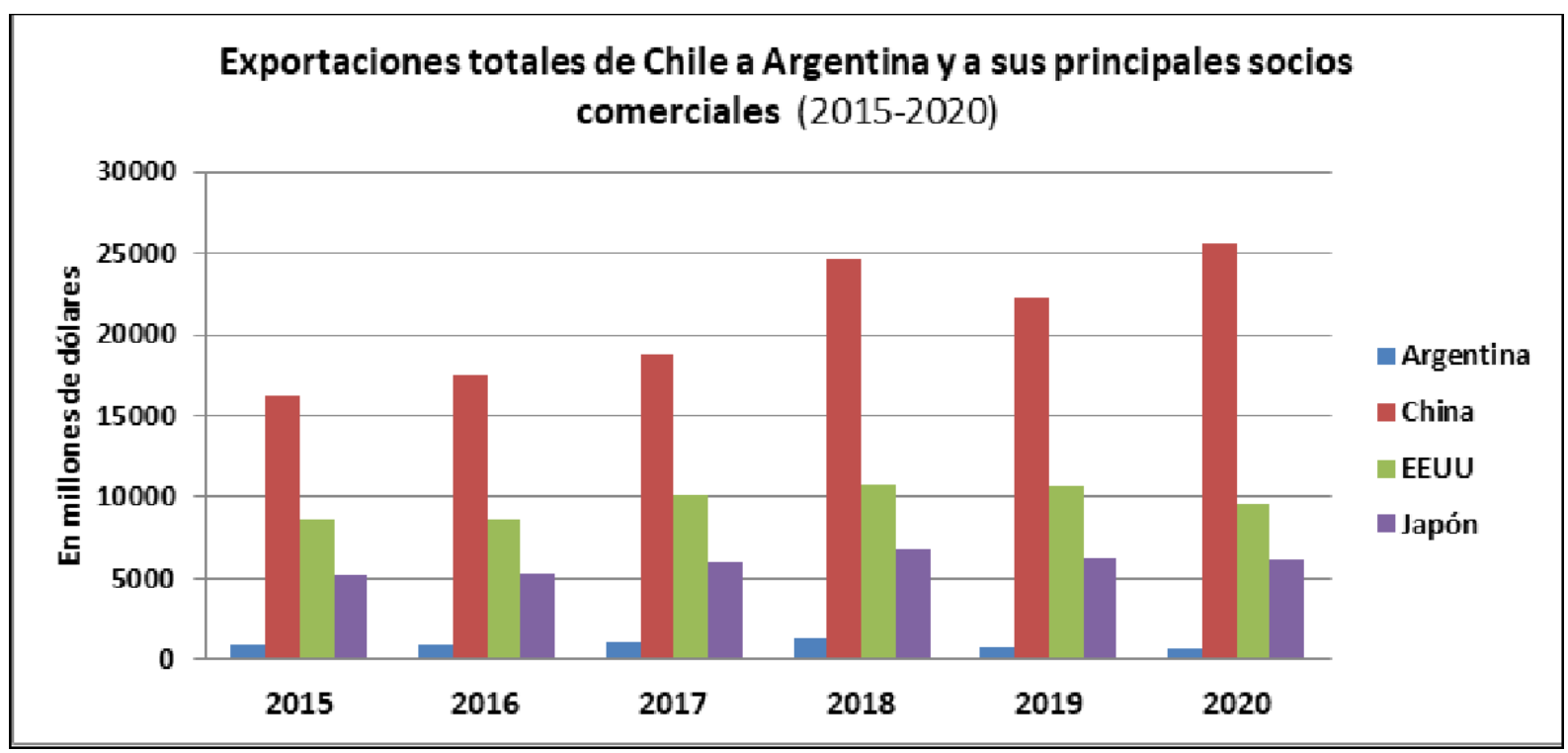

Fuente: Servicio Nacional de Aduana del Gobierno de Chile

Por último, es necesario observar cómo se componen las exportaciones que ambos países destinan a su contraparte. En lo que respecta a Argentina, el Gráfico 4 demuestra los principales rubros de su canasta exportadora hacia Chile, como así también las fluctuaciones que sufrieron los mismos durante el período 2015-2020.

En primer lugar, se destaca la presencia de los alimentos, que crecieron de 668 millones de dólares en 2015 a más de 1300 millones en 2020. Seguidamente, debe 
hacerse mención de los combustiblesy lubricantes minerales que aumentaron desde 191 millones hasta los 751 millones en 2019. En tercer lugar, se distinguen los rubros de medios de transporte y maquinaria, que fluctúan entre 156 y 372 millones, y entre los 72 y 85 millones respectivamente. De esta forma, puede verse el crecimiento constante que tuvieron las exportaciones argentinas hacia Chile en el periodo estudiado, exceptuando el año 2016 y el retroceso que sufrieron en 2020.

Es menester destacar la diversidad de productos que nuestro país exporta al mercado chileno. La presencia de manufacturas de origen industrial (MOI), si bien es pequeña en comparación con aquellas de origen agropecuario (MOA). Para un país donde la demanda de dólares es crónica esto posee una gran trascendencia. Asimismo, si se recuerda el superávit que sostiene Argentina con respecto a Chile, puede concluirse que la relación comercial con este último es sumamente beneficiosa y la única - entre sus principales socios comerciales- que no genera un déficit en su balanza comercial.

\section{Gráfico 4: Canasta exportadora de Argentina con destino a Chile}

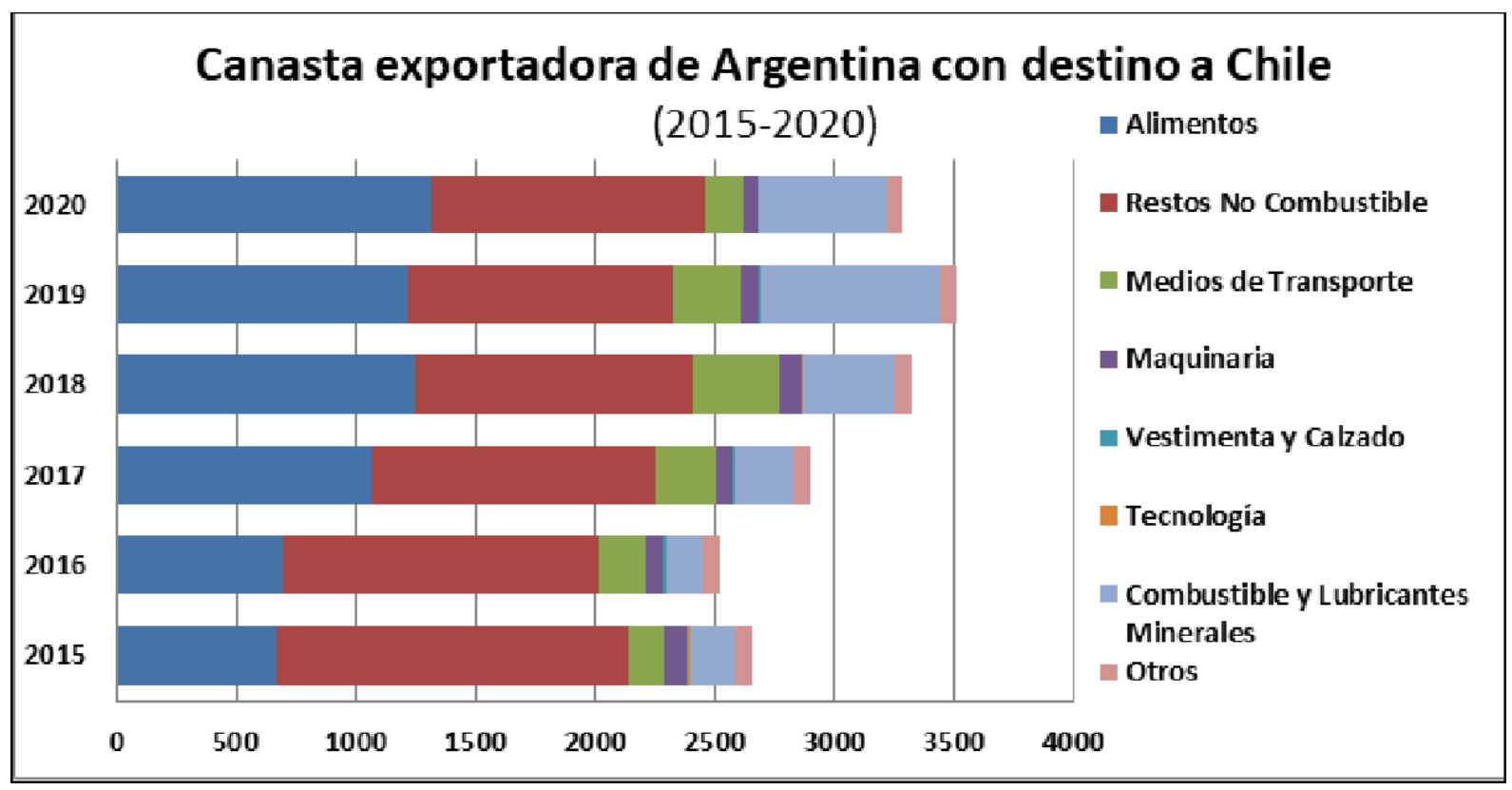

Fuente: Servicio Nacional de Aduana del Gobierno de Chile

Chile presenta sus particularidades demostradas en el Gráfico 5. Las exportaciones totales con destino a Argentina representaron menos de la mitad que las argentinas que ingresaron al mercado chileno. Se destaca el año 2018, al superar los 1200 millones de dólares. No obstante, en dicho año las exportaciones argentinas superaron los 3000 millones.

Sin tener en cuenta "restos no mineros", sobresalen los servicios al superar los 87 millones de dólares. En segundo lugar, se ubican los minerales superando los 70 millones de dólares pese a sufrir una fuerte retracción en 2019 y 2020. En tercer lugar, se ubica el rubro "forestales". Por último, deben mencionarse los productos de mar y las frutas. Como puede apreciarse, en el caso chileno las materias primas acaparan el mayor porcentaje, seguidas por los servicios. De esta manera, lo exportado por el país vecino 
no sólo arroja cifras muy inferiores a la de su contraparte, sino que se caracteriza por una menor diversificación. Finalmente, los datos recogidos demuestran cómo la pandemia no retrajo lo exportado por la nación chilena, ya que la fuerte caída se produjo en 2019.

\section{Gráfico 5: Canasta exportadora de Chile con destino a Argentina}

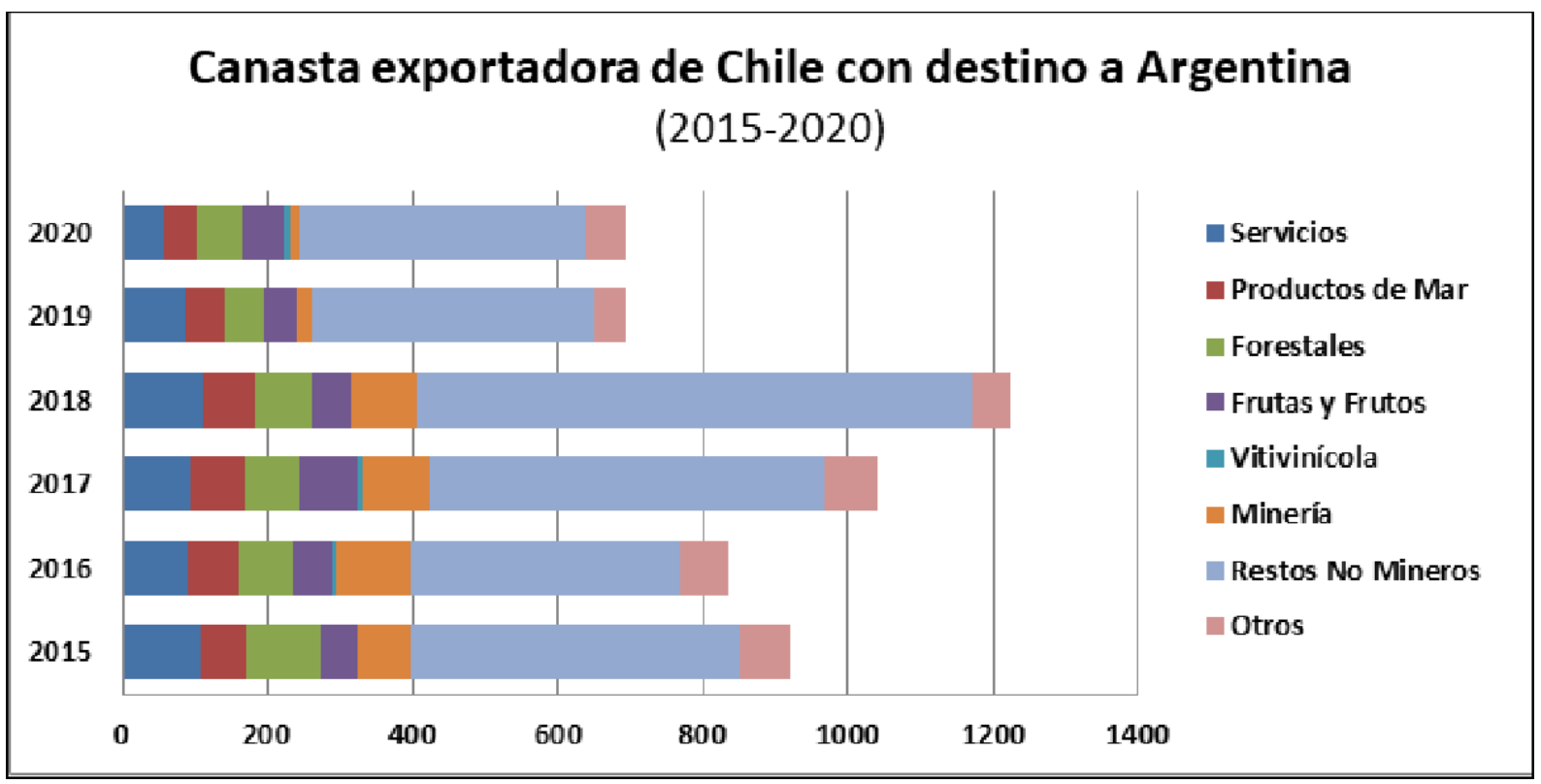

Fuente: Servicio Nacional de Aduana del Gobierno de Chile

\section{El escenario posterior a la vigencia del acuerdo}

Sobrevino la pandemia y tras quince meses de entrada en vigor, se realizó el primer encuentro virtual para aprobar los planes de trabajo de las diversas disciplinas que abarca el acuerdo.

Así, en agosto de 2020 se formalizó la I Reunión de la Comisión Administradora Bilateral del Acuerdo Comercial entre Argentina y Chile. El apremiante contexto de pandemia propició tratar con urgencia la simplificación, modernización y armonización de los procedimientos de exportación e importación a través de la implementación de herramientas digitales como la ventanilla única de comercio exterior. Y, en este mismo orden de importancia, también se fijó una hoja de ruta atendiendo el desarrollo de la economía digital en tiempos de pandemia para impulsar el reconocimiento de la firma digital entre ambos países.

Asimismo, en esta reunión se aprobaron los mecanismos instituidos para abordar bilateralmente las "Medidas Sanitarias y Fitosanitarias" (capítulo 4) y los "Obstáculos Técnicos al Comercio" (capítulo 5). Otro de los temas que enfatizaron ambas partes fue el capítulo 7 de "Contrataciones Públicas" que permitirá el acceso de proveedores de Argentina y Chile, con prioridad para las MIPyMEs, a las licitaciones públicas de ambos países. 
En materia "Género y Comercio" se diseñó un programa de trabajo que se desarrollará durante los próximos dos años con el objetivo de fortalecer el rol de la mujer en el sector empresario y el de incorporar la perspectiva de género en el intercambio bilateral. Recordemos que, en el caso de Argentina, se trata del primer instrumento de comercio internacional que incluye este tema.

Uno de los temas que se acordó fue incluido por la parte negociadora de Chile. Es un formato suficientemente promocionado por el país trasandino en todos sus frentes de negociaciones. Se tratade percibir a Chile como el país de salida de las exportaciones de América Latina aprovechando la red de Tratados de Libre Comercio (TLC) que tiene este país. De hecho, la Dirección General de Relaciones Económicas Internacionales (DIRECON) del Ministerio de Relaciones Exteriores de Chile, a través del Departamento Cadenas Globales de Valor (CGV) lleva adelante diversas iniciativas para explorar encadenamientos productivos con países de la región para la generación de nuevas oportunidades de negocios. En este sentido, uno de los consensos alcanzados fue la decisión de incorporar al Acuerdo Comercial un capítulo sobre Encadenamientos Productivos.

Se puede destacar dos instancias que se deducen de este acuerdo: la puesta en funcionamiento de la eliminación del cobro del servicio de roaming y el acuerdo dentro del sector automotor entre la Argentina y Chile- para exportar 10.000 unidades de vehículos por una suma que asciende a los 150 millones de dólares. Esta última negociación se encuentra, hasta el momento actual, aún en negociación.

\section{Reflexiones finales}

En mayo de 2019, momento de la entrada en vigencia del tratado comercial aquí analizado, pocos podían imaginar los sucesos que vendrían en los meses subsiguientes. El cambio de gobierno en Argentina del partido opositor a la administración que negoció el acuerdo. Las masivas movilizaciones que irrumpieron en Santiago y que promovieron la reforma constitucional chilena. La pandemia que puso en jaque al mundo entero. A pesar de este escenario, las relaciones bilaterales entre Argentina y Chile lograron dinamismo, incluso en contextos de comunicaciones ambiguas entre Sebastián Piñera y el nuevo mandatario Alberto Fernández.

La vigencia del acuerdo es muy reciente y la retracción global del comercio producto de los avatares del coronavirus- provocó que los datos actuales en materia de exportación e importación no presenten variaciones importantes. Sin embargo, puede decirse que para concretar un estudio más exhaustivo del impacto económico del acuerdo comercial hace falta generar mecanismo que contabilicen las nuevas disposiciones del acuerdo. Especialmente, se requiere afrontar mayores esfuerzos por parte de ambos países para cuantificar las transacciones de servicios (capítulo 9) y de comercio electrónico (capítulo 11). Con la falta de estas herramientas y sinergia de las cuentas nacionales se dificulta el análisis de los efectos que pueda llegar a tener la implementación de este instrumento.

Lo anterior también impacta en la descomposición y el examen de los encadenamientos productivos para evaluar en qué momento, bajo qué modalidad y a qué costos (si es que resulta más beneficioso) se sumará Argentina con sus economías 
regionales a las cadenas de valor chilenas. En esta misma línea, es preciso configurar un esquema de consulta y mesas de negociación con los gobiernos subnacionales y el sector privado para estimar qué posibilidades hay de avanzar en asociaciones con distintos actores económicos.

Asimismo, se cree oportuno avanzar en nuevos canales de diálogo entre las Cancillerías de Argentina y Chile con los gobiernos regionales y provinciales para avanzar en iniciativas de coordinación, cooperación, convergencia e integración con el alcance que abarca las temáticas del acuerdo. El proceso de descentralización en Chile es el escenario y fundamento propicio para esto.

En definitiva, la relación comercial no cambió en términos cuantitativos tras el acuerdo. No obstante, es un hito que se enmarca en una relación bilateral institucionalizada y dinámica que tiene mucha capacidad y oportunidad para potenciarse a través de los círculos concéntricos.

\section{Bibliografía}

Actis, E. y Creus, N (2020). La disputa por el poder global. Rosario, Argentina. Capital Intelectual.

Acuerdo de Complementación Económica N 16. República Argentina y República de Chile (12 de septiembre de 1996). Disponible en repositorio de ALADI: https://www.subrei.gob.cl/docs/default-source/acuerdos/argentina/acuerdo-decomplmentaci\%C3\%B3n-econ\%C3\%B3mica-ace-n--16.pdf?sfvrsn=a9d9edc6_2

Baldwin, R. (2011). 21st century regionalism: Filling the gap between 21st century trade and 20th century trade rules, N. ESRD-2011-08, Organización Mundial del Comercio. Disponible: http://dx.doi.org/10.30875/c67646a3-en

Colacrai, M. (2016). La relación bilateral Argentina-Chile en clave política 2010-2015. Continuidades y matices con cambios de gobierno; Revista Estudios Internacionales 183 - ISSN 0716-0240. Instituto de Estudios Internacionales - Universidad de Chile. Santiago, Chile.

Declaración VIII Reunión Binacional de Ministros de Argentina y Chile (16 de diciembre 2016). Disponible:

http://www.sice.oas.org/TPD/ARG_CHL/12_2016_VIII_Ronda_Declaracion_s.pdf

Instituto Nacional de Estadísticas y Censo (IPEC) (2021). Informe Técnico del Intercambio Comercial Argentino. República Argentina. Disponible en:

https://www.indec.gob.ar/indec/web/Nivel4-Tema-3-2-40

Nieto, N. (2019). Argentina y Chile en la Antártida: ¿cooperación o conflicto latente? (2000-2018). Repositorio de la Universidad Nacional de Rosario. Disponible en https://rephip.unr.edu.ar/handle/2133/17307

Sexagésimo Primer Protocolo Adicional (2 de noviembre de 2017). Disponible en: https://www.subrei.gob.cl/docs/default-source/acuerdos/argentina/texto-acuerdo-delibre-com ercio-chile-argentina.pdf?sfvrsn=da8b6d7_2 
Revista Integración y Cooperación Internacional, no 34, enero-junio 2022, pp. 49-64

ISSN 1852-9798

Servicio Nacional de Aduana del Gobierno (2021). Estadísticas de Comercio Exterior. República de Chile. Disponible en:

https://www.aduana.cl/aduana/site/edic/base/port/estadisticas.html?filtro=20181205220 $\underline{946}$

NIETO, N. y SACCO, I. (2022). Argentina, Chile y un acuerdo de última generación. Revista Integración y Cooperación Internacional, 34 (ene-jun), 49-64 